\title{
Aspectos éticos de la gestión académica en una Facultad de Medicina
}

\section{IGNACIO SÁNCHEZ D.}

This article states that the incorporation of ethical issues such as bioethics and principles of good practice, facilitates the academic management in a Faculty of Medicine. It presents some interesting aspects necessary to carry out a quality academic management that serves the university mission and its educational and research project. It underlines good practices regarding not only professional relations but also organizational aspects. It includes an analysis of academic management and its association with quality and leadership, it reflects on the ethical values of quality work and on the theory of functional or interactional leadership, a key element to boost the development of a university health institution.

(Rev Med Chile 2011; 139: 1097-1102).

Key words: Bioethics; Education, medical; Leadership.

Departamento de Pediatría, Facultad de Medicina, Pontificia Universidad Católica de Chile. Recibido el 19 de octubre de 2010, aceptado el 4 de mayo de 2011.

Correspondencia a: Dr. Ignacio Sánchez D. Rector

Pontificia Universidad Católica de Chile. Av. Lib. Bernardo O'Higgins 340. Santiago, Chile. Teléfono: (56-2) 354-2346 Fax: (56-2) 354-2211 E-mail: igsan@med.puc.cl
E n la gestión académica de una Facultad de Medicina es importante destacar aspectos éticos que garanticen que el trabajo se oriente al servicio de la misión, del proyecto educativo y de la investigación que desarrolla la institución universitaria. El presente trabajo revisa algunos principios generales, el marco ético, consideraciones sobre la calidad de la gestión, y el tema central de liderazgo y su orientación al desarrollo académico. El objetivo de este trabajo es aportar elementos de discusión y análisis en un tema de real interés, orientado a realizar una mejor gestión académica.

\section{Los principios de la bioética}

El Informe Belmont distinguió tres principios éticos básicos: respeto por las personas, beneficencia y justicia. Debido a que este Informe sólo se refirió a las cuestiones éticas surgidas en el ámbito de la investigación clínica con seres humanos, Beauchamp y Childress ${ }^{1}$ reformularon estos principios para ser aplicados a la ética en el trabajo asistencial. Estos autores presentaron el orden histórico de aparición de estos principios: no maleficencia, beneficencia, autonomía y justicia. En forma breve, se describen estos principios: La no maleficencia hace referencia a la obligación de no infringir daño intencionadamente. Este principio se inscribe en la tradición de la máxima clásica primum non nocere ("lo primero no dañar"). La beneficencia consiste en prevenir el daño, eliminarlo o hacer el bien; puede entenderse como todo tipo de acción que tiene por finalidad el bien de otras personas. La autonomía es un concepto que admite grados, es respetada cuando se le reconoce el derecho a mantener puntos de vista, a tomar decisiones y a realizar acciones basadas en valores y creencias personales, y la justicia consiste en "dar a cada uno lo suyo". De un modo similar, se entiende que la justicia es el tratamiento equitativo y apropiado a la luz de lo que es debido a una persona. Estos cuatro principios resumen los valores básicos de la bioética y están en concordancia con fundamentos filosóficos y antropológicos.

\section{Marco ético para la gestión académica}

Se denomina gestión a una actividad organizada dirigida a la consecución de un bien social, 
un bien propio o básico. El concepto de buenas prácticas se refiere al conjunto de principios, valores, conductas y hábitos, así como a los procesos y estructuras organizativas que permiten alcanzar el fin o bien social propuesto. Por esto, las buenas prácticas y la adecuada gestión persiguen convertir en hábito el "hacer las cosas bien", tanto en el nivel individual como en la comunidad ${ }^{2}$. En el caso de una comunidad académica, la situación presenta características especiales, ya que al estar constituida por pares (profesores, alumnos, funcionarios administrativos), es muy importante resaltar los elementos de formación, coherencia y valores para poder implementar un marco de convivencia ética y social acorde a una adecuada gestión académica. En este sentido, la capacitación y perfeccionamiento de sus líderes y autoridades es prioritaria, y debe realizarse con un apropiado plan de educación continua. La Figura 1 presenta un modelo que puede reflejar la estructura de las conductas en una comunidad académica ${ }^{3}$.

Los códigos de comportamiento éticos se refieren normalmente a los valores que guían nuestra conducta y que orientan nuestra actuación como miembros de una organización. Los valores morales constituyen orientaciones generales que guían nuestra conducta en una dirección determinada. Dentro de este sistema se encuentran valores como dignidad, respeto, responsabilidad, cuidado, igualdad, justicia e integridad. En el caso del trabajo en una comunidad universitaria, estos valores se encuentran sistematizados en forma global en los llamados principios básicos de la ética del trabajo.

En forma general, se puede decir que las normas representan la cristalización por parte de una sociedad de determinados valores. Mientras que los valores orientan, las normas obligan. A diferencia de las normas tradicionales y las jurídicas, vinculadas siempre a una determinada comunidad o estado y bajo mecanismos externos de sanción, las normas morales se identifican como tales precisamente por su universalidad y la autodeterminación a seguirlas. Su carácter vinculante deriva de la conformidad o aceptación por parte de las personas como miembros de una comunidad a la que se pertenece por el sólo hecho de ser un individuo con igualdad de derechos y deberes. La Tabla 1 describe las diferentes normas que nos rigen como sociedad ${ }^{3}$.

Una guía de buenas prácticas se entiende así como el conjunto de conductas y procedimientos que nos permiten en forma más adecuada alcanzar el fin social que legitima la actividad en cuestión. De ahí que sea necesario un paso más en la orientación hacia una conducta ética que recoja la conjunción entre valores, como parte de nuestra forma de ser. Este es el caso cuando se vive en una Institución que tiene una orientación iluminada por la Fe, en cuyo caso el marco global de trabajo debe estar irradiado por todos los elementos valóricos y de opción de trabajo que implique el proyecto educacional y universitario. Esta condición no significa sesgo, o falta de apertura sino sólo explicita una profunda orientación y convicción, la que debe quedar establecida en forma explícita y ser coherente con el plan de desarrollo propuesto. La identidad, la apertura y el discernimiento son los criterios básicos de trabajo al interior de una universidad que busca el diálogo fe-razón.

Se propone que la gestión de una institución universitaria se realice desde la perspectiva de servir de vehículo para lograr la excelencia, entendida como se ha señalado en el anhelo por mejorar lo bueno en términos de profesionalización, humanización y calidad. Se asume que la organización universitaria y todos sus integrantes o partes interesadas forman una comunidad que apela a mínimos morales o principios de justicia cuando

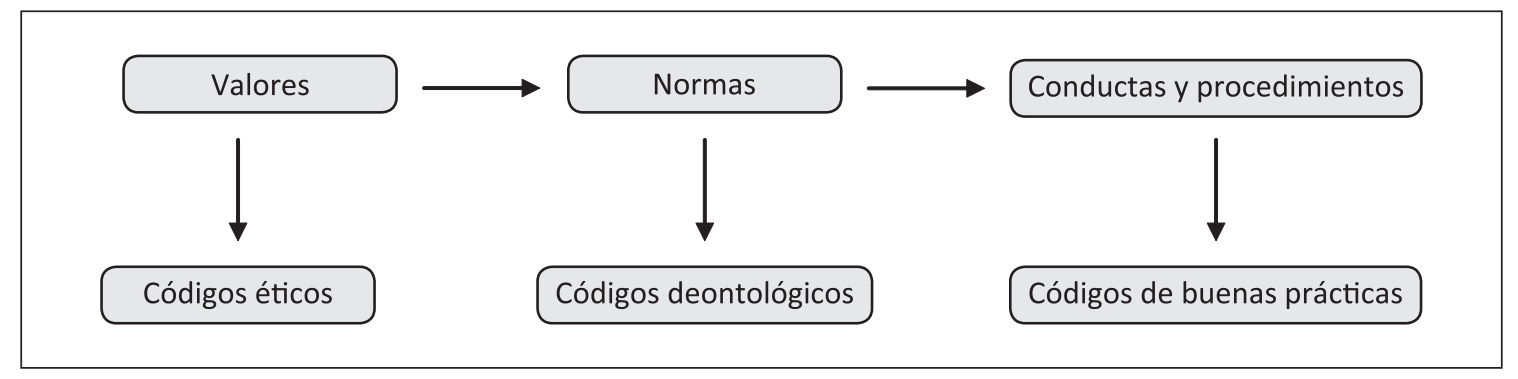

Figura 1. La estructura de la toma de decisiones y de la acción (Modificado de García Marzá, D. Ref. 3). 
Tabla 1. Tipos de normas que se establecen en una Institución

\begin{tabular}{|ll|}
\hline Norma & Descripción \\
Jurídicas & $\begin{array}{l}\text { Dirigidas a los ciudadanos, su motivación es externa, acompaña de la fuerza de coacción del Estado. Son } \\
\text { muy importantes para el logro de la sana convivencia en comunidad }\end{array}$ \\
\hline Tradicionales & Dirigidas a los miembros de una comunidad o cultura, su obligación es extensa \\
Morales & $\begin{array}{l}\text { Dirigidas a todas las personas como seres humanos, su motivación es interna: nuestro propio convenci- } \\
\text { miento }\end{array}$ \\
Religiosas & $\begin{array}{l}\text { Dirigidas a los miembros de una comunidad religiosa, la obligación es interna y está relacionada a las } \\
\text { creencias compartidas }\end{array}$ \\
\hline
\end{tabular}

(Adaptado de García Marzá, D. Ref. 3).

evalúa las decisiones y prácticas. Es en este punto donde es crucial establecer los elementos básicos de las normas de sana convivencia: verdad, lealtad, franqueza, respeto, valores éticos y comunicación efectiva. De esta forma se puede lograr tener una cultura de respeto, honestidad y confianza, que permita enfrentar grandes desafíos y superar obstáculos en común, convencidos de que se es parte de una comunidad con una Misión y metas comunes $^{4-6}$.

Esta metodología de gestión posee dos etapas que conviene diferenciar analíticamente, aunque en el día a día de la gestión se den de modo interrelacionado.

La etapa descriptiva: la comunidad académica se entiende como un grupo de personas con diferentes intereses que poseen valor intrínseco o legítimo y a los que debe dar satisfacción, con comunicación y defensa de sus intereses, y la etapa estratégica: una vez definidos los grupos de participantes es necesario tomar decisiones referentes a qué se debe hacer, cómo se debe actuar con cada una de estas relaciones. Al definir las estrategias se tiene en consideración que existen expectativas o intereses por parte de los diferentes estamentos que pueden poseer un valor inherente, que son legítimos y a los que la organización debe satisfacer. En este punto, debemos reconocer que toda organización tiene una cultura propia, y una Universidad con mayor razón. Los cambios que planifican los que están a cargo de la gestión académica, deben considerar en detalle lo que existe, respetarlo y considerarlo. En este sentido, con respeto y conociendo la historia, se deben hacer los cambios de forma y fondo en la gestión, de acuerdo a la misión y orientación de la institución ${ }^{7-9}$.

En el caso de la salud, una definición de buena práctica sanitaria, que está relacionada a la gestión en la línea de lo expuesto hasta el momento, nos la ofrece la Organización Mundial de la Salud (1994), cuando afirma que: "la buena práctica es un proceso que persigue una mejora continua en su realización en la gestión y dirección de la salud, el entorno y la seguridad involucrando a todos los actores involucrados dentro y fuera de la institución".

La definición de buenas prácticas -tanto clínicas como organizacionales- resulta de vital importancia para afrontar los desafíos de las organizaciones de educación superior, especialmente aquéllas que poseen una gestión privada dentro del sistema público.

\section{Gestión académica y calidad}

Ética y calidad están claramente relacionadas y habría que decir que la ética comprende uno de los aspectos de la calidad al tener el compromiso de realizar el trabajo con esfuerzo y dedicación. En este punto se debe reflexionar sobre los valores éticos del trabajo de calidad. El profesional y, en especial, el que trabaja en gestión académica, debe hacer su mejor esfuerzo por trabajar con calidad y tener siempre en su plan de trabajo el concepto de hacer una entrega al máximo de sus capacidades, como sentido de responsabilidad, de los valores y fortalezas individuales y también -y quizá más importante aún- como una manera de hacer un testimonio de servicio al prójimo, desde el lugar de trabajo en que cada uno se desempeña. En este aspecto, el concepto debe ser: a mayor capacidad y oportunidades, mayor obligación de servicio y entrega a la sociedad y a cada uno de sus individuos. Los criterios y estándares vinculados al 
principio de justicia son quizá los más difíciles de evidenciar, pero sin duda son todos aquellos que hacen referencia a los modelos de enfocar la gestión de la organización desde la perspectiva ética de la justicia ${ }^{10}$.

Para ello, es necesario dar el paso de una bioética centrada en los aspectos de ética clínica a una denominada social, ya que debe afrontar tanto la promoción de la ética institucional, como el control desde la ética social de las políticas públicas de salud y de la evolución del propio sistema de salud, así como de las relaciones y adecuación entre el sistema y las exigencias y necesidades de la sociedad.

La calidad de la atención conlleva varios aspectos: excelencia en cuanto a obtener los mejores resultados, valor en cuanto al precio de los servicios ofrecidos, ajuste a unas determinadas especificaciones o estándares, y satisfacción de las expectativas de los usuarios. Se requiere también una medicina mucho más cercana al paciente, con una relación más personal, más humanizada, tanto por parte del médico y demás profesionales de la salud, como por parte de la institución, por ejemplo, con planes concretos de humanización de la atención que se presta. La Tabla 2 presenta el impacto de las acciones en la Ética clínica, institucional y social ${ }^{8}$.

Es importante destacar el principio de solidaridad, más allá de la justicia, tanto a nivel social como individual. Una sociedad puede implementar un sistema que generalice la asistencia en salud a todos, con prestaciones de alta calidad si lo permite el desarrollo económico, pero podría ser calificado como deshumanizado, distante, no acogedor, por parte de los usuarios, y puede dar lugar a un aumento de las quejas o a una mala percepción de la atención recibida.

\section{Gestión académica y liderazgo}

En las diferentes organizaciones, las decisiones de las personas que se ubican en niveles más altos de la jerarquía constituyen el marco de referencia y las premisas para la toma de decisiones. La posibilidad de que las autoridades transmitan la misión y los planes de desarrollo es clave al momento de lograr una unidad en la acción. La teoría funcional del liderazgo expone que el rol del líder está intervinculado con los roles de los seguidores y que, como todo rol, las relaciones se plantean en

Tabla 2. Sistema de Principios en Bioética. Se presenta el impacto en la Bioética clínica, institucional y social

\begin{tabular}{|lll|}
\hline $\begin{array}{l}\text { Bioética clínica } \\
\text { Médico-paciente }\end{array}$ & $\begin{array}{l}\text { Bioética institucional } \\
\text { Institución-profesional-usuario }\end{array}$ & $\begin{array}{l}\text { Bioética social } \\
\text { Instituciones-Sociedad }\end{array}$ \\
\hline $\begin{array}{c}\text { No Maleficencia } \\
\text { - Deber de no abandono }\end{array}$ & - No abandono institucional & - No abandono social \\
- Precaución & - Precaución: sistemas seguridad & - Prevención salud \\
- Responsabilidad profesional y familiar & - Responsabilidad Institución & - Responsabilidad política \\
Justicia & & \\
- Respeto derechos paciente & - Respeto derechos pacientes & - Promoción derechos \\
- Equidad & - Equidad & - Seguimiento inequidades \\
- Eficiencia y continuidad & - Eficiencia institucional & - Control, acreditación \\
Autonomía & & \\
- Respeto autonomía & - Participación pacientes y familia & - Control social \\
- Promover competencia & - Educación en salud & - Participación comunidad \\
- Confidencialidad & - Confidencialidad & - Prevención/educación \\
& & - Confidencialidad \\
Beneficencia & & \\
- Calidad de vida & - Calidad asistencial & - Calidad sistema \\
- Excelencia profesional & - Excelencia institucional & - Acreditación \\
- Humanización cuidado & - Humanización institucional & - Subsidiariedad \\
- Ética del cuidado & - Solidaridad institucional & \\
\hline
\end{tabular}

(Modificado de F.J. León, ref. 8). 
términos de expectativas. La consecuencia que se desprende es que para entender el liderazgo en un grupo hay que considerar tanto al líder como al resto de los componentes de la estructura gru$\mathrm{pal}^{11}$. En la Tabla 3, se presentan dos situaciones en que se enfrenta la realidad de una comunidad o empresa: los equipos que van en ascenso o en declive. Es importante notar que la capacidad de autocrítica, franqueza y comunicación, junto a la coherencia del líder y de su equipo de trabajo, son claves para el éxito del proyecto.

En la actualidad se plantea una teoría ecléctica del liderazgo. Es conocida como teoría interaccional y señala, brevemente, que el liderazgo es el resultado de la interacción entre cuatro variables: a) la estructura de personalidad del líder; b) las personalidades de los seguidores; c) la estructura y función del grupo, y d) la situación por la que atraviesa el grupo.

Esta teoría tiene gran importancia en organizaciones en que se busca conseguir niveles crecientes de compromiso de parte de los subalternos, dado que muestra la importancia de la gradualidad y la estrecha correspondencia entre líder y seguidores. El liderazgo es, por consiguiente, un rol que ha de ser asumido y que implica obligaciones y derechos para quien lo sustenta. El líder, al respecto goza de gran poder e influencia para conseguir modificar las normas grupales; pero, no obstante, al mismo tiempo es quien más debe regirse por ellas. Dado que las contravenciones a las normas son castigadas normalmente con pérdida de status, el líder es quien más tiene que perder. Además, ninguna alteración a las normas grupales puede ser más dañina que la ejecutada por el propio líder. En las organizaciones formales, coexisten habitualmente dos tipos de poder: a) el poder formal: delegado, impersonal, específico, referido a puestos, delegable, y b) el liderazgo: poder informal, personalizado, emergente, espontáneo, no específico y no delegable ${ }^{11}$.

Para las organizaciones resulta mucho más manejable, planificable y predecible el poder que emerge de las bases. En la Tabla 4 se expresan algunas características necesarias para poder ejercer un liderazgo eficaz en una institución. El carácter académico, le imprime aún más el sello de la coherencia y de poder lograr la autoridad, por consenso y por el peso específico del trabajo realizado.

Para finalizar, cabe destacar la importancia del trabajo bien realizado, el que se realiza con dedicación, coherencia, verdad, equidad y justi-

Tabla 3. Dinámicas de conducta de un líder y su equipo

\begin{tabular}{|ll|}
\hline \multicolumn{2}{|c|}{ La forma en que interactúan los ejecutivos dice mucho acerca de la situación de la empresa } \\
\hline Equipos en declive & Equipos en ascenso \\
\hline Se blinda a quienes tienen el poder & Las personas presentan las situaciones para discutirlas \\
\hline $\begin{array}{l}\text { Se hacen valer opiniones sin entregar información, evidencia } \\
\text { o argumentos sólidos que las respalden }\end{array}$ & $\begin{array}{l}\text { Las personas aportan datos, evidencia, lógica y argumentos } \\
\text { sólidos a la discusión }\end{array}$ \\
$\begin{array}{l}\text { El líder cuestiona muy poco los argumentos, evitando de este } \\
\text { modo las críticas }\end{array}$ & $\begin{array}{l}\text { El líder emplea un estilo socrático, cuestionando y desafiando } \\
\text { a las personas para lograr entendimientos sólidos }\end{array}$ \\
$\begin{array}{ll}\text { Los miembros del equipo aceptan una decisión pero no tra- } \\
\text { bajan en forma unida por el éxito de la misma }\end{array}$ & $\begin{array}{l}\text { Los miembros del equipo se unen tras una decisión, luego } \\
\text { trabajan por su éxito }\end{array}$ \\
$\begin{array}{l}\text { Los miembros del equipo buscan obtener el mayor crédito } \\
\text { posible para sí mismos, pero no cuentan con la admiración y y } \\
\text { confianza de sus pares }\end{array}$ & $\begin{array}{l}\text { Cada uno de los miembros del equipo da crédito a los otros } \\
\text { por logrado, y cuenta con la confianza y admiración }\end{array}$ \\
$\begin{array}{ll}\text { Los miembros del equipo discuten y expresan sus puntos } \\
\text { de vista }\end{array}$ & $\begin{array}{l}\text { Los miembros del equipo discuten y debaten para encontrar } \\
\text { las mejores respuestas que apoyen la causa general }\end{array}$ \\
$\begin{array}{ll}\text { El equipo conduce "autopsias" buscando responsables en en } \\
\text { lugar de enseñanzas }\end{array}$ & $\begin{array}{l}\text { El equipo conduce "autopsias", obteniendo enseñanzas a } \\
\text { partir de las experiencias dolorosas }\end{array}$ \\
$\begin{array}{l}\text { Los miembros del equipo no suelen generar resultados ex- } \\
\text { traordinarios y culpan a otros o a factores externos por sus } \\
\text { errores, incumplimientos y fracasos }\end{array}$ & $\begin{array}{l}\text { Cada uno de los miembros del equipo genera resultados } \\
\text { excenales, e incluso en el caso de un error, aceptan la }\end{array}$ \\
\hline
\end{tabular}

(Modificado de Collins J. Ref. 11). 
Tabla 4. Características necesarias para ejercer un liderazgo eficaz

\begin{tabular}{|l|l|}
\hline Características de un líder \\
- & Plantea la Misión y luego realiza la acción \\
- & Se preocupa de las personas y apoya \\
- & Compromete e irradia al grupo \\
- & Está orientado a la verdad \\
- Posterga los deseos personales, da el ejemplo \\
- Considera los principios y los grandes temas \\
- Planifica y actúa, no sólo entrega ideas \\
- No ocupa demasiado tiempo en el plan \\
- Incorpora y es leal con los colaboradores \\
- Acepta a las personas, no intenta cambiarlas \\
- Trabaja con humildad, es auténtico \\
- Sabe esperar, es paciente \\
- La popularidad no es lo importante \\
- Aplica el sentido común al analizar los problemas \\
- Admite errores, es flexible
\end{tabular}

cia, para incorporar los elementos éticos que son necesarios par un trabajo de gestión académica de excelencia. Para citar a Gómez-Lobo, se puede decir que "el elemento clave que hace que el trabajo (y el estudio) sea un bien humano, es la experiencia de logro y autorrealización que constituye su núcleo central"12. Al no ser posible la mantención de un trabajo aislado y solitario, tenemos un acercamiento personal con el prójimo, por lo tanto, debemos considerarlo un facilitador de las relaciones interpersonales. Uno de los elementos más importantes en el trabajo y, en especial, en este tema de la gestión académica, es lograr percibir lo que la escritora Pearl Buck describe como que "en el trabajo se debe perseguir la extraordinaria sensación que da el hecho de hacer algo bien". Al enfrentar de esta forma nuestra diaria tarea, estaremos cumpliendo con la misión que se le entrega a los que ejercen liderazgo en la gestión académica.

\section{Referencias}

1. Beauchamp TL, Childress JF. Principios de ética biomédica. Barcelona: Masson; 1999.

2. Conill J. Horizontes de economía ética. Aristóteles, Adam Smith, Amartya Sen. Madrid: Tecnos, 2006.

3. García Marzá, D. La apuesta ética en las organizaciones sanitarias. Castellón, España: Universitat Jaume I. (2005).

4. Couceiro A, Beca JP. Los Comités de ética Asistencial y las repercusiones jurídicas de sus informes. Rev Med Chile 2006; 134: 517-9.

5. Gracia D. Prólogo. En: Beaucham T, Childress JF. Principios de ética biomédica. Barcelona: Masson, 1999: 9-15.

6. Simón P. Ética de las organizaciones sanitarias. Nuevos modelos de calidad. Madrid: Triacastela, 2005.

7. León FJ. Diez años de Bioética en América Latina: historia reciente y retos actuales. En: Lolas F ed. Diálogo y cooperación en salud. Diez años de Bioética en la OPS. Santiago: OPS-OMS, 2004; 145-52.

8. León FJ. De la Bioética clínica a una Bioética Institucional y Social. En: Editorial Ad Hoc. Cuadernos de Bioética. Buenos Aires, 2008; 21-38.

9. Tauber A. Patient Autonomy and the Ethics of Responsibility. Massachusetts: MIT Press, 2005.

10. Rodríguez, D. Gestión Organizacional. Elementos para su estudio. Santiago de Chile: Ediciones Universidad Católica de Chile, 2007.

11. Collins J. The Dynamics of Leadership-Team Behavior. Business Week 2009; 28-32.

12. Gómez-Lobo A. Los bienes humanos. Ética de la ley natural. Santiago de Chile: Mediterráneo; 2006. 